\title{
Effects of Maternal Anxiety Disorders on Infant Self-Comforting Behaviors: The Role of Maternal Bonding, Infant Gender and Age
}

\author{
Mitho Müller ${ }^{\mathrm{a}}$ Ed Tronick ${ }^{\mathrm{d}} \quad$ Anna-Lena Zietlow $^{\mathrm{b}} \quad$ Nora Nonnenmacher $^{\mathrm{b}}$ \\ Stephan Verschoor ${ }^{a}$ Birgit Träuble ${ }^{c}$ \\ ${ }^{a}$ Department of Psychology, Ludwig Maximilian University Munich, Munich, ${ }^{b}$ Heidelberg University Hospital,

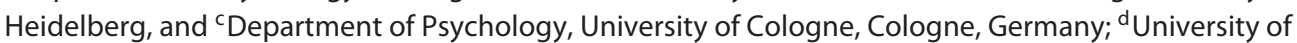 \\ Massachusetts Boston and Harvard Medical School, Boston, Mass., USA
}

\section{Key Words \\ Maternal anxiety disorders - Maternal bonding - Infant self-comforting behaviors - Face-to-Face Still-Face paradigm . Gender differences in infancy}

\begin{abstract}
Background/Aims: We investigated the links between maternal bonding, maternal anxiety disorders, and infant selfcomforting behaviors. Furthermore, we looked at the moderating roles of infant gender and age. Methods: Our sample $(n=69)$ comprised 28 mothers with an anxiety disorder (according to DSM-IV criteria) and 41 controls, each with their 2.5- to 8-month-old infant ( 41 females and 28 males). Infant behaviors were recorded during the Face-to-Face Still-Face paradigm. Maternal bonding was assessed by the Postpartum Bonding Questionnaire. Results: Conditional process analyses revealed that lower maternal bonding partially mediated between maternal anxiety disorders and increased self-comforting behaviors but only in older female infants (over 5.5 months of age). However, considering maternal anxiety disorders without the influence of bonding, older female infants (over 5.5 months of age) showed decreased rates of self-comforting behaviors, while younger male infants (under 3 months of age) showed increased rates in the
\end{abstract}

case of maternal anxiety disorder. Conclusions: The results suggest that older female infants (over 5.5 months of age) are more sensitive to lower maternal bonding in the context of maternal anxiety disorders. Furthermore, results suggest a different use of self-directed regulation strategies for male and female infants of mothers with anxiety disorders and low bonding, depending on infant age. The results are discussed in the light of gender-specific developmental trajectories.

c) 2016 S. Karger AG, Basel

\section{Introduction}

Maternal bonding refers to the emotional ties that mothers develop towards their infants during the first weeks after childbirth [1]. Impaired bonding can result in rejection, hostility, aggressive impulses towards the child $[2,3]$, an increased risk of abuse and neglect [4], and even infanticide $[5,6]$. Therefore, maternal bonding is considered a critical process for ensuring maternal care and thus infant development [4].

\section{Mitho Müller and Ed Tronick share first authorship.}


Several postpartum disorders have been hypothesized to have a negative effect on maternal bonding. For instance, a large body of literature has consistently shown postpartum (subclinical) depression to pose a significant risk for maternal bonding [7-12]. Although the prevalence of postpartum anxiety disorders $(11.1 \%)$ is almost twice as high as that of postpartum depression (6.1\%) [13], very few studies have systematically examined the effects of maternal anxiety on maternal bonding. Evidence supporting the claim that postpartum anxiety disorders adversely affect mothers' emotional bonding was recently found by Tietz et al. [14] in a prior evaluation of the current data set. A Bangladeshi study reported corroborating evidence [15]; maternal anxiety symptoms correlated negatively with mothers' emotional bonding. Likewise, Dubber et al. [16] found significant associations between anxiety measures and maternal bonding.

Previous research on risk factors for infant development found a negative impact of maternal depression [17, $18]$ and anxiety $[19,20]$ and has focused on maternal sensitivity [21, 22] and mother-infant interaction [23, 24]. Recently, Dalsant et al. [25] demonstrated that parental bonding moderated later stress response to social stimuli in the adult offspring. Those that experienced optimal maternal bonding during childhood showed calmer stress responses to distressing stimuli, while less optimal bonding led to increased stress responses. Although such studies suggest that maternal bonding affects stress regulation later in life, they do not shed any light on the immediate consequences for stress regulation during infancy.

Caregivers are thought to play an important role in the development of stress regulation in infants. Sensitive reactions towards the child are hypothesized to provide an important external source of stress regulation [26-28]. Indeed, higher maternal sensitivity (attention as well as prompt and appropriate reaction to infants' signals) is associated with increased behavioral and physiological regulation [29], less negative temperament [30], and emotional resilience at older ages [31]. However, due to time constraints and other factors caregivers cannot be attentive to their infants all the time nor can they always respond sensitively, even when attentive. When caregivers are temporarily inattentive and infants experience the need for external stress regulation, they can signal the caregiver for attention (e.g. by a caregiver-directed gaze, reaching out) by 3 months of age. If such other-directed regulatory behaviors [32] fail, infants engage in self-directed stress regulation $[26,32]$ such as hand-to-mouth movements and nonnutritive sucking to reduce stress [28].
Usually, these self-comforting behaviors decrease with age as infants engage in more complex regulatory strategies. Cole et al. [33] assume a developmental sequence in which infants first have a basic self-regulatory capacity of varying and limited effectiveness, then engage with caregivers for external stress regulation, and finally develop an array of additional self-regulatory strategies over the toddler and preschool years. An exaggerated and prolonged dependence on self-directed stress regulation may have aversive effects. For instance, Braungart-Rieker et al. [34] found that higher levels of self-directed stress regulation at 4 months of age predicts insecure-avoidant attachment at 1 year of age. Koulomzin et al. [35] found a comparable result. They investigated infant behaviors at 4 months of age in later insecure-avoidant and secure infants. They concluded that self-directed stress regulation facilitates behavioral coordination in insensitive caregiving environments. Yet they discussed that a strong reliance on self-directed stress regulation may increase the risk for persistent emotional difficulties. This may be due to disengagement to opportunities to externally regulate stress [35] and limited effectiveness of self-directed stress regulation in more demanding situations [36]. Accordingly, a study by Müller et al. [37] found that self-directed stress regulation is linked to increased salivary cortisol reactivity, which leads to the conclusion that self-comforting behavior can be seen as an indicator of increased stress.

Low maternal bonding might represent an insensitive caregiving environment. Noorlander et al. [38] found lower maternal bonding to be negatively associated with behaviors that are indicative of sensitive caregiving. Since Tietz et al. [14] found that postpartum anxiety disorders adversely affect mothers' emotional bonding, infants of caregivers with postpartum anxiety disorders might be less able to externally regulate the infants' stress due to low maternal bonding. Furthermore, a direct effect of maternal depression on infants' regulatory strategy has been reported. Manian and Bornstein [39] observed that infants of clinically depressed mothers use more self-directed stress regulation compared to controls. Though these findings refer to maternal depression, maternal anxiety disorders might analogously represent an insensitive caretaking environment. Some studies found that anxious mothers demonstrate more insensitive and less sensitive behaviors compared to controls [e.g. 40, 41]. These findings suggest that insensitive caretaking, due to maternal anxiety disorders and low maternal bonding, may lead to an increased self-directed stress regulation. We therefore hypothesized that maternal anxiety disor- 
ders are associated with increased infant self-comforting behaviors during the mother-infant interaction and that this association is mediated by lowered maternal bonding.

We expected these effects to be dependent on two variables: infant gender and age. The following findings support this hypothesis: male infants are known to have greater difficulties than female infants in maintaining interactive regulation, and mother-son dyads take longer to repair interactive errors [42]. Furthermore, male infants are more affected by maternal depressive symptoms than female infants, and mother-son dyads have a less welladapted interaction than mother-daughter dyads with highly depressive mothers [43]. Moreover, male infants may be more sensitive to distress due to differences in hormonal function and cortisol responses [44]. We therefore hypothesized that male infants would show more self-comforting behaviors than female infants in response to maternal anxiety disorders and low maternal bonding. The second hypothesized moderator is infant age. It is well known that self-regulatory strategies change with age [34]. However, Tronick [26] argues that the self-directed regulatory style might be perpetuated due to the experience of chronic failure to externally regulate stress. This line of argument predicts that older infants that have experienced insensitive caregivers should show increased self-comforting behaviors. We therefore expected older infants to show more self-comforting behaviors than younger infants if their mothers report lower bonding and are diagnosed with anxiety disorders. To examine our hypotheses, we assessed maternal bonding in mothers with and without anxiety disorders in the postpartum period and infant self-directed regulatory behaviors in their 2.5- to 8-month-old infants. We used the Face-toFace Still-Face paradigm (FFSF) to assess infants' self-directed regulation to the stress of the experimental interruption of maternal engagement $[45,46]$.

\section{Materials and Methods}

Sample

The sample $(\mathrm{n}=69)$ is part of a larger longitudinal study [14, $37,47]$. Subjects were recruited between June 2006 and October 2010. Assessments took place at the University Hospital of Heidelberg, Germany. Inclusion criteria for women of the clinical group were at least one of the following anxiety disorders according to the DSM-IV [48] in the postpartum period: panic disorder with agoraphobia, agoraphobia without history of panic disorder, generalized anxiety disorder, social phobia, obsessive compulsive disorder, posttraumatic stress disorder, and anxiety disorder not otherwise specified. Exclusion criteria for patients included: an acute
Table 1. Diagnostic status of the clinical sample $(n=28)$

n $(\%)$

Disorders

Panic disorder/agoraphobia

Social phobia

$0(35.7)$

Specific phobia

Obsessive-compulsive disorder

$4(14.3)$

Posttraumatic stress disorder

$13(46.4)$

Generalized anxiety disorder

$1(3.6)$

Anxiety disorder NOS

$17(60.7)$

$4(14.3)$

$1(3.6)$

Comorbid binge eating disorder

$1(3.6)$ Onset

Before pregnancy

$16(57.1)$

During pregnancy

$5(17.9)$

Postpartum

$7(25.0)$

Number of anxiety disorders

One

$13(46.4)$

Two

$8(28.6)$

Three or more

$7(25.0)$

Percentages were calculated relative to the sample size of the clinical group. Due to comorbidity between anxiety disorders, there were more diagnoses than participants.

or former psychosis, a current or former bipolar disorder, current substance abuse, acute suicidal tendency, or acute major depression or dysthymia. Healthy controls needed to have no current or antecedent axis I diagnosis according to the DSM-IV. Initially, 122 mothers with their infants were recruited for the larger study. We excluded 20 mothers who met the diagnostic exclusion criteria from the analyses. An additional 13 mothers entered the study at a later study point. Interactive measures of 11 dyads were missing, and 9 mothers did not fill out questionnaires. Consequently, 28 women with an anxiety disorder and 41 control women and their 2.5- to 8-month-old infants were included in the analyses. Maternal anxiety disorders are summarized in table 1 . Full demographic statistics and group comparisons are summarized in table 2 .

\section{Procedures}

The full study procedure is described by Müller et al. [37]. The study protocol was approved by the independent ethics committee of the Heidelberg University Medical Faculty. Written informed consent was obtained after the study procedures had been fully explained to the mothers. Mother-infant interaction during the FFSF was videotaped in our laboratory. It consisted of three episodes each lasting $2 \mathrm{~min}$ : first, an initial face-to-face interaction in which the mothers are instructed to play with their infant as usual (without the aid of toys and pacifiers). Next, the still-face episode in which the mothers have to turn their head aside while silently counting to ten and then turn back to the infant but not engage in any gestures, facial expressions, or vocalizations. Finally, the procedure ends with the reunion episode in which the mother is required to resume face-to-face play with her infant. Subsequently, the Structured Clinical Interview-I for DSM-IV Disorders 
Table 2. Maternal and infant demographics and tests on comparability of subgroups

\begin{tabular}{|c|c|c|c|c|c|c|c|}
\hline & General & Control & Anxiety & $\mathrm{t}(\mathrm{p})$ & Female & Male & $\mathrm{t}(\mathrm{p})$ \\
\hline Infant age, months ${ }^{\mathrm{a}}$ & $4.1 \pm 1.5$ & $3.8 \pm 1.3$ & $4.7 \pm 1.6$ & $2.76(0.01)$ & $4.3 \pm 1.6$ & $3.9 \pm 1.3$ & $1.27(0.21)$ \\
\hline Gestation age, weeks ${ }^{\mathrm{b}}$ & $39.5 \pm 1.4$ & $39.7 \pm 1.3$ & $39.3 \pm 1.7$ & $1.08(0.28)$ & $39.7 \pm 1.4$ & $39.2 \pm 1.5$ & $1.42(0.16)$ \\
\hline APGAR (average) ${ }^{c}$ & $9.5 \pm 0.6$ & $9.6 \pm 0.6$ & $9.5 \pm 0.5$ & $0.56(0.58)$ & $9.5 \pm 0.6$ & $9.5 \pm 0.5$ & $0.27(0.79)$ \\
\hline Infant gender & General & Control & Anxiety & $\chi^{2}(\mathrm{p})$ & Female & Male & $\chi^{2}(\mathrm{p})$ \\
\hline Female infants & 41 & 23 & 18 & $0.46^{\mathrm{e}}(0.50)$ & - & - & - \\
\hline \multirow[t]{2}{*}{ Male infants } & 28 & 18 & 10 & & - & - & - \\
\hline & General & Control & Anxiety & $\mathrm{t}(\mathrm{p})$ & Female & Male & $t(p)$ \\
\hline Maternal age, years ${ }^{\mathrm{d}}$ & $33.2 \pm 5.4$ & $33.5 \pm 5.5$ & $32.8 \pm 5.2$ & $0.56(0.58)$ & $33.2 \pm 5.0$ & $33.2 \pm 5.9$ & $0.03(0.974)$ \\
\hline Maternal education & General & Control & Anxiety & $\mathrm{U}(\mathrm{p})$ & Female & Male & $\mathrm{U}(\mathrm{p})$ \\
\hline University degree & 38 & 25 & 13 & $494.0(0.28)$ & 22 & 16 & $507.5(0.37)$ \\
\hline University entrance qualification & 16 & 8 & 8 & & 7 & 9 & \\
\hline High secondary qualification & 13 & 7 & 6 & & 10 & 3 & \\
\hline Low secondary qualification & 2 & 1 & 1 & & 2 & 0 & \\
\hline Marital status & General & Control & Anxiety & $\chi^{2}(\mathrm{p})$ & Female & Male & $\chi^{2}(\mathrm{p})$ \\
\hline Married & 47 & 29 & 18 & $0.79^{f}(0.37)$ & 27 & 20 & $50.33^{\mathrm{g}}(0.56)$ \\
\hline Not married & 20 & 10 & 10 & & 13 & 7 & \\
\hline
\end{tabular}

Values are means \pm SD or numbers, as appropriate, unless otherwise indicated.

${ }^{\mathrm{a}} \min =2.5 ; \max =7.9 .{ }^{\mathrm{b}} \min =36.3 ; \max =41.9 .{ }^{\mathrm{c}} \min =7.0 ; \max =10.0 .{ }^{\mathrm{d}} \min =23.0 ; \max =45.0 .^{\mathrm{e}} 0$ cells have expected count $<5$, minimum expected count is 11.36 . ${ }^{\mathrm{f}} 0$ cells have expected count $<5$, minimum expected count is 8.36 . ${ }^{\mathrm{g}} 0$ cells have expected count $<5$, minimum expected count is 8.06 .

(SKID-I) [50], was carried out. Finally, the German version of the Postpartum Bonding Questionnaire (PBQ-16) [8] was filled out at home.

\section{Measures}

Structured Clinical Interview for DSM-IV Axis I Disorders. Postpartum maternal anxiety was assessed with the German version of SKID-I [50]. The SKID-I is a widely used semistructured interview for the diagnosis of selected axis I disorders.

German Version of Postpartum Bonding Questionnaire. The PBQ was originally developed by Brockington et al. $[10,12]$ as a self-reporting screening tool for impaired bonding. We used the German abridged 16-item version of the PBQ suggesting a singlefactor solution [8]. The PBQ-16 had an internal consistency of Cronbach's $\alpha=0.87$ in our sample. Higher values indicate more impaired bonding. The sum score ranges from 0 to 80 .

Coding of Infant Behavior during the FFSF: Infant and Caregiver Engagement Phases-Revision. Full information on the Infant and Caregiver Engagement Phases-Revision (ICEP-R) is described by Müller et al. [37]. We coded the videotapes using the Noldus Observer Video-Pro ${ }^{\circledR}$ coding system with 1-second time intervals. Infants' and mothers' behavior during the FFSF was coded by two trained and reliable coders using the German translation and revision of the microanalytical ICEP-R [50]. The ICEP-R combines information from the infant's and caregiver's face, direction of gaze, and vocalizations. Additionally, for infants and in accordance with the maternal and infant engagement codes [e.g. as described in 37], oral and manual self-comforting behaviors were coded. Oral self-comforting included (1) the infants' initiated skin contact between their own body parts and their mouth, (2) the infants' initiated mouth contact to exogenous objects or (3) sucking on the caregiver's hand or fingers (self-initiated or not). Manual self-comforting behaviors were coded if the infants touched one hand with the other. The primary independent measures were the sum of relative time proportions of infant self-comforting behaviors, that is, the sum of seconds in which infants engage in oral or manual self-comforting behaviors divided by the time of the FFSF [37]. Thus, the scale ranges from 0 to 1 . Descriptive results are multiplied by $100 \%$.

The coders were blinded to the hypotheses of the study and the maternal psychiatric status; $31 \%$ of the videotapes (28 dyads of 90 videotapes) were randomly selected and coded by two independent study coders. They were not aware which videos were used for assessing coding reliability. In accordance with other studies $[24,51]$, we used Cohen's $\kappa$ [52] to compute interrater reliability. This was $\kappa=0.73$ for the infant codes and $\kappa=0.78$ for the maternal 
Fig. 1. PROCESS - Model 76. Path a: direct effect of maternal anxiety disorder on maternal bonding moderated by infant gender and age. Path $\mathrm{a}^{*} \mathrm{~b}$ : indirect effect of maternal anxiety disorder on infant self-comforting behaviors mediated by maternal bonding and moderated by infant gender and age. Path c: direct effect of maternal anxiety disorder moderated by infant gender and age.

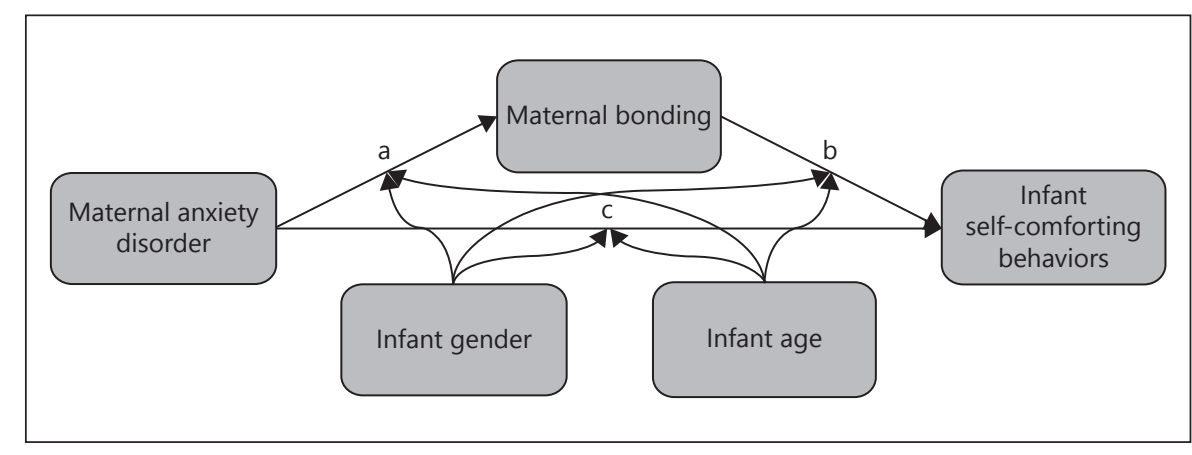

codes. Thus, the interrater reliability of our study coders was similar to those reported in previous studies $[24,51]$.

Data Analyses

We used the Statistical Package for Social Sciences (IBM ${ }^{\mathrm{TM}}$ SPSS $^{\circledR}$ v. 23.0.0.0) for all analyses conducted. Power estimates were computed using G-Power v. 3.1.9.2 [53, 54]. To ensure that we did not take out a specific group of the study population by the case exclusions as described in the sample section, we carried out Little's Missing Completely at Random (MCAR) test [55]. The MCAR test evaluates if the MCAR condition is fulfilled. If nonsignificant, differences between excluded cases and the remaining sample are unlikely. In addition, missing values are unlikely to depend on third variables. For the MCAR test, we considered the following variables: sociodemographic data (e.g. age), interaction variables (ICEP-R), maternal bonding (PBQ-16), and data assessed at birth (e.g. gestation age), as well as questionnaire data regarding psychopathology (not described here; e.g. the State-Trait Anxiety Inventory, STAI [56]). Furthermore, differences concerning maternal age, gestation age, APGAR values, infant age, maternal education, and marital status between controls and their clinical counterparts and between males and females were explored (via t tests, $U$ tests, and $\chi^{2}$ tests) to ensure comparability between the groups.

We were interested to see whether bonding mediates a main effect of maternal anxiety disorder on infant self-comforting behaviors and whether infant gender and age play moderating roles for this mediation. We carried out conditional process analyses - a regression-based approach - using the SPSS ${ }^{\circledR}$ Makro 'PROCESS' (v. 2.15) [57]. We tested the model depicted in figure 1. It estimates the indirect effect of maternal anxiety disorder on infant self-comforting behaviors mediated by maternal bonding and moderated by infant gender and age (fig. 1 , path $\mathrm{a}^{*} \mathrm{~b}$; table 5 ). This estimation is based on the direct effect of maternal anxiety disorder on maternal bonding moderated by infant gender and age (fig. 1, path a; table 4, model 1) and the direct effects of study variables on infant self-comforting behaviors (as demonstrated in table 4, model 2). Moreover, the direct effect of maternal anxiety disorder on infant self-comforting behaviors moderated by infant gender and age (fig. 1, path c; table 6) is estimated in the same model. The standard errors and confidence intervals of the indirect (mediated) effects are bootstrapped and bias corrected ( $\mathrm{n}=1,000$ samples). Variables were mean centered. Empirical p values are two-tailed (critical $\alpha=$ 0.05 ). The conditional process analysis requires a single measurement outcome. Thus, we aggregated infant self-comforting behaviors over the FFSF episodes.

Effects of Maternal Anxiety Disorders on Infant Self-Comforting Behaviors
Table 3. Means and standard errors of infant self-comforting behaviors and maternal bonding by maternal diagnoses and infant gender

\begin{tabular}{lcr}
\hline & $\begin{array}{l}\text { Self-comforting } \\
\text { behavior }\end{array}$ & $\begin{array}{c}\text { Maternal } \\
\text { bonding }\end{array}$ \\
\hline Control & $13.2(3.1)$ & $6.8(1.1)$ \\
Anxiety & $16.0(3.8)$ & $12.5(1.4)$ \\
Female & $14.6(3.0)$ & $9.1(1.1)$ \\
Male & $14.6(3.7)$ & $10.2(1.3)$ \\
Control female & $19.0(4.0)$ & $7.1(1.4)$ \\
Control male & $7.4(4.6)$ & $6.6(1.6)$ \\
Anxiety female & $10.2(4.6)$ & $11.0(1.6)$ \\
Anxiety male & $21.9(6.0)$ & $13.9(2.1)$ \\
\hline
\end{tabular}

Covariates appearing in the model are evaluated at infant age of 4.2 months.

\section{Results}

\section{Preliminary Analyses}

The MCAR test was nonsignificant $\left(\chi^{2}=207.31\right.$, d.f. $=190, p=0.19)$; the case exclusions were valid for our sample, and the subpopulation was representative of the larger sample. As demonstrated in table 2 only infant age differed between the clinical group and controls, revealing the control group to have younger infants. We controlled for infant age as the moderator in our analyses. Means and standard errors of infant self-comforting behaviors and maternal bonding by maternal diagnosis and infant gender adjusted for infant age are summarized in table 3 .

\section{Main Analysis}

Maternal Anxiety Disorder and Bonding. At first, path a (fig. 1) is estimated. As demonstrated in table 4 (model 1 ), there was a significant effect of maternal anxiety dis- 
Table 4. Direct effects of study variables and their interaction terms on maternal bonding and infant self-comforting behaviors

\begin{tabular}{|c|c|c|c|c|c|c|}
\hline \multicolumn{7}{|l|}{ Model outcome: maternal bonding ${ }^{\mathrm{a}}$} \\
\hline Anxiety disorder & 5.291 & 1.746 & 3.031 & 0.004 & 1.803 & 8.780 \\
\hline Infant gender & 0.867 & 1.674 & 0.518 & 0.606 & -2.478 & 4.211 \\
\hline Anxiety disorder $\times$ infant gender & 3.751 & 3.435 & 1.092 & 0.279 & -3.113 & 10.615 \\
\hline \multicolumn{7}{|l|}{ Model outcome: infant self-comforting } \\
\hline Maternal bonding & 0.006 & 0.004 & 1.784 & 0.080 & -0.001 & 0.013 \\
\hline Anxiety disorder & -0.013 & 0.052 & -0.256 & 0.799 & -0.118 & 0.091 \\
\hline Infant gender & -0.027 & 0.046 & -0.594 & 0.555 & -0.120 & 0.065 \\
\hline Infant age & 0.005 & 0.017 & 0.280 & 0.781 & -0.030 & 0.039 \\
\hline \multicolumn{7}{|c|}{$\begin{array}{l}\mathrm{SE}=\text { standard error. } \\
{ }^{\mathrm{a}} \text { Constant: } \mathrm{B}=-0.147, \mathrm{SE}=0.855, \mathrm{t}=-0.172, \mathrm{p}=0.864, \mathrm{CI}=-1.855,1.562 .{ }^{\mathrm{b}} \text { Constant: } \mathrm{B}=0.159, \mathrm{SE}=0.023, \mathrm{t}=6.790, \mathrm{p}<0.01 \text {, } \\
=0.112,0.206 .\end{array}$} \\
\hline
\end{tabular}

Table 5. Conditional indirect effects of anxiety disorder on infant self-comforting behaviors at values of the moderators

\begin{tabular}{llllll}
\hline $\begin{array}{l}\text { Infant } \\
\text { gender }\end{array}$ & $\begin{array}{l}\text { Infant age, } \\
\text { months }\end{array}$ & B & SE & $\begin{array}{l}\text { 95\% CI } \\
\text { lower limit }\end{array}$ & $\begin{array}{l}95 \% \mathrm{CI} \\
\text { upper limit }\end{array}$ \\
\hline Female & 2.7 & 0.021 & 0.036 & -0.026 & 0.142 \\
& 4.1 & 0.035 & 0.028 & -0.001 & 0.118 \\
& 5.6 & 0.050 & 0.040 & 0.001 & 0.172 \\
\hline Male & 2.7 & 0.008 & 0.066 & -0.122 & 0.154 \\
& 4.1 & 0.013 & 0.071 & -0.134 & 0.162 \\
& 5.6 & 0.018 & 0.093 & -0.156 & 0.235 \\
\hline
\end{tabular}

$\mathrm{SE}=$ Standard error

order on maternal bonding $(\mathrm{p}<0.01)$ : if mothers had an anxiety disorder, they reported lower bonding (an increase of 5.3 PBQ scale points on average in comparison to controls). There were no main or interaction effects of infant gender or age ( $\mathrm{p}>0.20)$.

Study Variables and Infant Self-Comforting Behaviors. Next, as the second basis for estimations of direct and indirect effects of maternal anxiety disorder on infant selfcomforting behaviors, all direct effects of the study variables and their two-way interaction terms were estimated (table 4, model 2). There was only a trend for a direct independent effect of maternal bonding on infant self-comforting behaviors $(\mathrm{p}=0.08)$ : the lower the maternal bonding, the higher the rate of infant self-comforting behaviors. Furthermore, there was a significant independent interaction between anxiety disorder and infant gender $(p=0.04)$. There were no direct independent effects of anxiety disorder, infant gender, and age, nor were there further interaction effects on infant self-comforting behaviors ( $\mathrm{p}>0.10)$.

Bonding as a Moderated Mediator between Maternal Anxiety Disorders and Infant Self-Comforting Behaviors. Table 5 summarizes all conditional indirect (mediated) effects of maternal anxiety disorder on infant self-comforting behaviors for values of the moderators (fig. 1, path $\left.a^{*} b\right)$. To investigate the moderation by infant age, the effects were tested at three levels of infant age (mean \pm SD). We found a mediation of the effect of maternal anxiety disorder on infant self-comforting behaviors through maternal bonding for female infants at 5.6 months of age $(95 \% \mathrm{CI}=0.001,0.172)$ : maternal anxiety disorder increased the rates of infant self-comforting behaviors through maternal bonding by $5 \%$ for female infants at 5.6 months on average. There was no such effect for male infants. 
Table 6. Conditional direct effects of anxiety disorder on infant self-comforting behaviors at values of the moderators

\begin{tabular}{|c|c|c|c|c|c|c|c|}
\hline \multirow[t]{3}{*}{ Female } & 2.7 & -0.017 & 0.090 & -0.190 & 0.850 & -0.196 & 0.162 \\
\hline & 4.1 & -0.110 & 0.063 & -1.749 & 0.086 & -0.235 & 0.016 \\
\hline & 5.6 & -0.202 & 0.078 & -2.604 & 0.012 & -0.357 & -0.047 \\
\hline Male & 2.7 & 0.220 & 0.097 & 2.268 & 0.027 & 0.026 & 0.414 \\
\hline
\end{tabular}

$\mathrm{SE}=$ Standard error

The Moderating Role of Infant Age and Gender on the Direct Effect of Maternal Anxiety Disorders

Table 6 summarizes all conditional direct (moderated) effects of maternal anxiety disorder on infant self-comforting behaviors at values of the moderators (fig. 1, path c). For females at 4.1 months of age, there was a trend for an effect of maternal anxiety disorder on infant self-comforting behaviors $(\mathrm{p}=0.09)$. However, it was the opposite of the expected direction. Female infants of mothers with anxiety disorders at 4.1 months showed $11 \%$ lower levels of self-comforting behaviors on average compared to controls. This relation was the same and significant for female infants at 5.6 months of age ( $p=0.01,20 \%$ decreased rates of self-comforting behaviors). For male infants at 2.6 months of age, the effect was in the expected direction $(\mathrm{p}=0.03)$. Male infants of mothers with an anxiety disorder showed an increased rate of self-comforting behaviors by $22 \%$ on average in comparison to controls. There were no effects for older male infants $(p>0.16)$.

The power to find a large effect $\left(f^{2}=0.35\right)$ for single regression coefficients was $1-\beta=0.99$, for medium-sized effects $\left(f^{2}=0.15\right)$ it was 0.89 and for small effects $\left(f^{2}=\right.$ 0.02 ) it was 0.21 . Thus, in the case of nonsignificant results, only large and medium-sized effects could sufficiently be ruled out. We were not able to rule out small effects.

\section{Discussion}

We hypothesized that maternal anxiety disorders are associated with increased infant self-comforting behaviors during the FFSF and that this association is mediated by maternal bonding. Furthermore, we expected that these effects were moderated by the infants' gender and age. Clearly, the nature of the findings is complex as they relate to the interplay between anxiety, bonding, infant gender, and age, thus making straightforward interpretation difficult. Nonetheless, it is clear that each of these factors affect infant self-comforting regulatory behaviors.

Results on path a (fig. 1) indicate that mothers with an anxiety disorder report lower bonding. This finding is in accordance with studies suggesting that anxiety accounts for lower bonding $[15,16]$ and represents a methodological invariance and robustness of prior analyses on the current data [14].

Results on path $\mathrm{a}^{*} \mathrm{~b}$ (fig. 1 ) suggest that maternal anxiety disorders account for increased rates of self-comforting behaviors in older female infants (over 5.5 months of age) through lowered maternal bonding. This finding is in line with studies showing that maternal disorders increase self-directed stress regulation at 5 months of age [39]. It further corroborates the argument by Koulomzin et al. [35] that self-directed regulative behavior (at 4 months of age) is increased in insensitive raising environments, as low maternal bonding was shown to be associated with insensitive caregiving [38]. One explanation might be that anxious mothers who feel low bonding to their infant are less able to sensitively regulate the infant's distress. However, mediational analysis for maternal sensitivity was beyond the scope of the study and should be targeted in future research.

Nevertheless, it was unexpected that female infants are sensitive to low maternal bonding in the context of maternal anxiety disorders and males are not. To the best of the authors' knowledge, there are no other studies reporting similar results. One explanation for our finding might be that our gender-specific hypotheses are mainly based on research investigating expressive engagement behaviors such as displays of positive and negative affect [43]. 
In contrast, infant self-comforting behaviors are not characterized as negative or positive expression but as inward-directed behaviors. Thus, the effects of anxiety and low bonding might unfold differently for self-directed stress regulation than they do for expressive behaviors. Another possible explanation relates to differences in sensitivity to social signals. Landerholm and Scriven [58], as well as Freedman [59], argue that female infants are more sensitive to social signals. These assumptions were supported by studies demonstrating that newborn and 12-month-old females show higher preferences for social stimuli than nonsocial stimuli in comparison to males $[60,61]$. As low maternal bonding is negatively associated with behaviors that are indicative of sensitive caregiving [38], it might be that female infants are more sensitive to lack of maternal care in the case of low maternal bonding. Consequently, it may be that only female infants of anxious mothers with low bonding might perpetuate the selfdirected regulatory style into later development [26]. The argument that a strong reliance on self-directed stress regulation may increase the risk for persistent emotional difficulties [35] and the well-established finding that females are more likely to have internalizing disorders later in development $[62,63]$ speak for that assumption. These ideas clearly require further investigations.

The findings on path c (fig. 1) suggest that male infants show increased rates of self-directed stress regulation behaviors as a direct effect of maternal anxiety disorder, but it only holds for younger males (under 3 months of age). If one accepts self-comforting behaviors as signs of distress [37], this is in line with findings from Weinberg et al. [43], who found maternal depressive symptoms negatively affecting dyadic regulation more in mother-son dyads than mother-daughter dyads at 3 months of age. However, the authors did not investigate this relationship in older infants. Conversely, the direct effect of maternal anxiety on infant self-comforting behaviors was contradictory to our hypotheses for older females (over 5.5 months of age): in the case of maternal anxiety disorders, older females showed lower rates of infant self-comforting behaviors when the mediating effect of maternal bonding was partialled out. We are not the first to encounter gender differences regarding regulatory strategies. For instance, Leeb and Rejskind [64] found that female infants aged 3-4 months made more eye contact interaction with unfamiliar female adults. Montirosso et al. [65] argued that infant eye contact may serve as a compensatory regulation strategy in preterm infants aged 6-9 months. It may be that female infants make more compensatory use of other-directed regulative strategy as op- posed to self-comforting behaviors in the case of maternal disorder. However, the answer to this question remains unclear as we did not analyze other-directed regulatory strategies.

\section{Limitations}

First, our clinical sample consisted of mothers with different anxiety disorders. Furthermore, subgroups of specific anxiety disorders were small, and many mothers (55.2\% of the clinical sample) had more than one anxiety disorder. Consequently, we were not able to identify the impact of specific anxiety disorders. According to the DSM-5 [66], obsessive compulsive disorders are no longer classified as anxiety disorders. Therefore, special attention needs to be paid to this psychological disorder with regard to mother-infant interaction in future research. However, only 3 women in our sample were diagnosed with a single obsessive-compulsive disorder, while 10 suffered from at least one additional anxiety disorder. Because of the very few cases with obsessive-compulsive disorder exclusively, it was impossible to test for intergroup differences. Almost $70 \%$ of the clinical sample had a history of depressive disorders (20 mothers had a lifetime major depression and 1 mother had a lifetime dysthymia). As sample sizes would have been too small, we were not able to differentiate between these subgroups. Consequently, we were not able to draw inferential conclusions about specific effects of anxiety disorders and additional lifetime depressive disorders on maternal bonding and infant self-comforting behaviors in our study.

A total of $75.9 \%$ of the clinical sample had a prepartum onset of anxiety disorders such that effects of fetal programming [67] cannot be excluded. A high proportion of academic degrees characterizes our sample. Thus, our results might not be generalizable to the overall population. The data assessment for the main finding was cross-sectional. Consequently, we cannot draw causal conclusions. Furthermore, our results have to be replicated in larger samples before they can be interpreted in terms of interventional applicability. We might have missed small effects due to power limitations and as subgroups were small considering the moderators of infant gender and age in our sample. Infant age was significantly higher in our clinical group. Thus, the accuracy of subgroup means for older infants of the control group and younger infants of the clinical group is limited. Consequently, the moderating role of infant age in our analyses has to be interpreted cautiously and should be confirmed by future studies. Furthermore, our regression analytic approach might be flawed by multicollinearity. The sug- 
gested ideas about the reasons and implications of our results should be reinvestigated in different, more homogeneous, and larger age groups or repeated assessments of the FFSF throughout and beyond the first year of life analyzing maternal behaviors as well as infant other-directed regulation behaviors such as gaze and social monitoring.

\section{Conclusion}

The findings emphasize gender- and age-specific differences in the effect of maternal anxiety on infant selfdirected regulation. Our results suggest different reactions in female infants to maternal anxiety disorder on the one hand (fig. 1, path c) and low maternal bonding in the context of anxiety disorders on the other hand (fig. 1, path $\left.a^{*} b\right)$. In the case of low bonding along with maternal anxiety disorder, female infants seem to develop a reliance on self-directed regulation (fig. 1, path b). By contrast, young male infants already engage in self-directed regulative strategies in the case of maternal anxiety (fig. 1, path c).

\section{Acknowledgments}

This study was funded by the German Research Foundation (DFG; study RE/2249 3-1), the National Institute of Mental Health (RO1MH45547 to E.T., principal investigator), NICHD (5R01HD050459 to E.T.), and NSF (0819839 to E.T.).

We would like to thank all the volunteers who participated in the mother-infant studies at the Heidelberg University Clinic and all colleagues who implemented the work for this study and article. We also want to thank our friends and relatives for supporting our work.

\section{Disclosure Statement}

All authors declare no conflicts of interest.

\section{References}

1 Klaus MH, Kennel JH, Klaus P: Bonding: building the foundations of secure attachment and independence. Reading, AddisonWesley, 1995.

2 Brockington I: Postpartum psychiatric disorders. Lancet 2004;363:303-310.

3 Kumar RC: 'Anybody's child': severe disorders of mother-to-infant bonding. Br J Psychiatry 1997;171:175-181.

4 Brockington I: Motherhood and Mental Health. New York, Oxford University Press, 1996.

5 Hornstein C, Hohm E, Trautmann-Villalba P: Die postpartale Bindungsstörung: eine Risikokonstellation für den Infantizid? Forens Psychiatr Psychol Kriminol 2009;3:3-10.

6 Hornstein C, Trautmann-Villalba P: Infantizid als Folge einer postpartalen Bindungsstörung. Nervenarzt 2007;78:580-583.

7 Reck C, Zietlow A, Müller M, Dubber S: Perceived parenting stress in the course of postpartum depression: the buffering effect of maternal bonding. Arch Womens Ment Health 2016;19:473-482.

8 Reck C, Klier, CM, Pabst K, Stehle E, Steffenelli U, Struben K, Backenstrass M: The German version of the Postpartum Bonding Instrument: psychometric properties and association with postpartum depression. Arch Womens Ment Health 2006;9:265-271.

9 Möhler E, Brunner R, Wiebel A, Reck C, Resch F: Maternal depressive symptoms in the postnatal period are associated with longterm impairment of mother-child bonding. Arch Womens Ment Health 2006;9:273-278.

Effects of Maternal Anxiety Disorders on Infant Self-Comforting Behaviors
10 Brockington IF, Fraser C, Wilson D: The Postpartum Bonding Questionnaire: a validation. Arch Womens Ment Health 2006;9:233242.

11 Loh C, Vostanis P: Perceived mother-infant relationship difficulties in postnatal depression. Infant Child Dev 2004;13:159-171.

12 Brockington IF, Oates J, George S, Turner D, Vostanis P, Sullivan M, Loh C, Murdoch C: A screening questionnaire for mother-infant bonding disorders. Arch Womens Ment Health 2001;3:133-140.

13 Reck C, Struben K, Backenstrass M, Stefenelli U, Reinig K, Fuchs T, Sohn C, Mundt C: Prevalence, onset and comorbidity of postpartum anxiety and depressive disorders. Acta Psychiatr Scand 2008;118:459468.

14 Tietz A, Zietlow A, Reck C: Maternal bonding in mothers with postpartum anxiety disorder: the crucial role of subclinical depressive symptoms and maternal avoidance behaviour. Arch Womens Ment Health 2014;17: 433-442.

15 Edhborg M, Nasreen H, Kabir ZN: Impact of postpartum depressive and anxiety symptoms on mothers' emotional tie to their infants 2-3 months postpartum: a populationbased study from rural Bangladesh. Arch Womens Ment Health 2011;14:307-316.

16 Dubber S, Reck C, Müller M, Gawlik S: Postpartum bonding: the role of perinatal depression, anxiety and maternal-fetal bonding during pregnancy. Arch Womens Ment Health 2015;18:187-195.
17 Murray L, Cooper P, Hipwell A: Mental health of parents caring for infants. Arch Womens Ment Health 2003;6:s71.

18 Koutra K, Chatzi L, Bagkeris M, Vassilaki M, Bitsios P, Kogevinas M: Antenatal and postnatal maternal mental health as determinants of infant neurodevelopment at 18 months of age in a mother-child cohort (Rhea Study) in Crete, Greece. Soc Psychiatry Psychiatr Epidemiol 2013;48:1335-1345.

19 Ibanez G, Bernard JY, Rondet C, Peyre H, Forhan A, Kaminski M, Saurel-Cubizolles M: Effects of antenatal maternal depression and anxiety on children's early cognitive development: a prospective cohort study. PLoS One 2015;10:e0135849.

20 Brouwers EPM, van Baar AL, Pop VJM: Maternal anxiety during pregnancy and subsequent infant development. Infant Behav Dev 2001;24:95-106.

21 Mills-Koonce WR, Propper CB, Barnett M: Poor infant soothability and later insecureambivalent attachment: developmental change in phenotypic markers of risk or two measures of the same construct? Infant Behav Dev 2012;35:215-225.

22 Mills-Koonce WR, Gariepy J, Sutton K, Cox MJ: Changes in maternal sensitivity across the first three years: are mothers from different attachment dyads differentially influenced by depressive symptomatology? Attach Hum Dev 2008;10:299-317.

23 Noe D, Schluckwerder S, Reck C: Influence of dyadic matching of affect on infant self-regulation. Psychopathology 2015;48:173-183. 
24 Reck C, Noe D, Stefenelli U, Fuchs T, Cenciotti F, Stehle E, Mundt C, Downing G, Tronick EZ: Interactive coordination of currently depressed inpatient mothers and their infants during the postpartum period. Infant Ment Health J 2011;32:542-562.

-25 Dalsant A, Truzzi A, Setoh P, Esposito G: Maternal bonding in childhood moderates autonomic responses to distress stimuli in adult males. Behav Brain Res 2015;292:428-431.

26 Tronick EZ: Emotions and emotional communication in infants. Am Psychol 1989;44: 112-119.

27 Spangler G, Schieche M, Ilg U, Maier U: Maternal sensitivity as an external organizer for biobehavioral regulation in infancy. Dev Psychobiol 1994;27:425-437.

28 Kopp CB: Regulation of distress and negative emotions: a developmental view. Dev Psychol 1989;25:343-354.

29 Conradt E, Ablow J: Infant physiological response to the still-face paradigm: contributions of maternal sensitivity and infants' early regulatory behavior. Infant Behav Dev 2010; 33:251-265.

- 30 Jonas W, Atkinson L, Steiner M, Meaney M, Wazana A, Fleming AS; The MAVAN Research Team: Breastfeeding and maternal sensitivity predict early infant temperament. Acta Paediatr 2015;104:678-686.

-31 Conway AM, McDonough SC: Emotional resilience in early childhood. Ann NY Acad Sci 2006; 1094:272-277.

32 Gianino A, Tronick EZ: The mutual regulation model: the infant's self and interactive regulation and coping and defensive capacities; in Field TM, McCabe PM, Schneiderman $\mathrm{N}$ (eds): Stress and Coping across Development. Hillsdale, Erlbaum, 1988, pp 47-68.

-33 Cole PM, Martin SE, Dennis TA: Emotion regulation as a scientific construct: methodological challenges and directions for child development research. Child Dev 2004;75: 317-333.

34 Braungart-Rieker JM, Garwood MM, Powers BP, Wang X: Parental sensitivity, infant affect, and affect regulation: predictors of later attachment. Child Dev 2001;72:252-270.

- 35 Koulomzin M, Beebe B, Anderson S, Jaffe J, Feldstein S, Crown C: Infant gaze, head, face and self-touch at 4 months differentiate secure vs. avoidant attachment at 1 year: a microanalytic approach. Attach Hum Dev 2002; 4:3-24.

-36 Diener ML, Mangelsdorf SC: Behavioral strategies for emotion regulation in toddlers: associations with maternal involvement and emotional expressions. Infant Behav Dev 1999;22:569-583.

- 37 Müller M, Zietlow A, Tronick E, Reck C: What dyadic reparation is meant to do: an association with infant cortisol reactivity. Psychopathology 2015;48:386-399.
38 Noorlander Y, Bergink V, van den Berg MP: Perceived and observed mother-child interaction at time of hospitalization and release in postpartum depression and psychosis. Arch Womens Ment Health 2008;11:49-56.

39 Manian N, Bornstein MH: Dynamics of emotion regulation in infants of clinically depressed and nondepressed mothers. J Child Psychol Psychiatry 2009;50:1410-1418.

40 Kaitz M, Maytal HR, Devor N, Bergman L, Mankuta D: Maternal anxiety, mother-infant interactions, and infants' response to challenge. Infant Behav Dev 2010;33:136-148.

41 Nicol-Harper R, Harvey AG, Stein A: Interactions between mothers and infants: impact of maternal anxiety. Infant Behav Dev 2007;30: 161-167.

42 Weinberg MK, Tronick EZ, Cohn JF, Olson KL: Gender differences in emotional expressivity and self-regulation during early infancy. Dev Psychol 1999;35:175-188.

43 Weinberg MK, Olson KL, Beeghly M, Tronick EZ: Making up is hard to do, especially for mothers with high levels of depressive symptoms and their infant sons. J Child Psychol Psychiatry 2006;47:670-683.

44 Elsmén E, Steen M, Hellström-Westas L: Sex and gender differences in newborn infants: why are boys at increased risk? J Mens Health Gend 2004;1:303-311.

45 Mesman J, van IJzendoorn MH, BakermansKranenburg MJ: The many faces of the StillFace Paradigm: a review and meta-analysis. Dev Rev 2009;29:120-162.

46 Adamson LB, Frick JE: The Still Face: a history of a shared experimental paradigm. Infancy 2003;4:451-473.

47 Reck C, Müller M, Tietz A, Möhler E: Infant distress to novelty is associated with maternal anxiety disorder and especially with maternal avoidance behavior. J Anxiety Dis 2013;27: 404-412.

48 Diagnostic and Statistical Manual of Mental Disorders: DSM-IV-TR, ed 4, text rev. Washington, American Psychiatric Association, 2000.

49 Wittchen H-U, Wunderlich U, Gruschwitz S, Zaudig M: SKID-I: Strukturiertes klinisches Interview für DSM-IV. Achse I. Psychische Störungen. Göttingen, Hogrefe, 1997.

50 Reck C, Noe D, Cenciotti F, Tronick E, Weinberg KM: Infant and Caregiver Engagement Phases, German revised ed (ICEP-R). Heidelberg, Universitätsklinikum Heidelberg, 2009.

51 Montirosso R, Provenzi L, Tavian D, Morandi F, Bonanomi A, Missaglia S, Tronick E, Borgatti R: Social stress regulation in 4-month-old infants: contribution of maternal social engagement and infants' 5-HTTLPR genotype. Early Hum Dev 2015;91:173179.

52 Cohen J: A coefficient of agreement for nominal scales. Educ Psychol Meas 1960;20:3746.
53 Faul F, Erdfelder E, Buchner A, Lang A: Statistical power analyses using G*Power 3.1: tests for correlation and regression analyses. Behav Res Methods 2009;41:1149-1160.

54 Faul F, Erdfelder E, Lang A, Buchner A: GPower 3: a flexible statistical power analysis program for the social, behavioral, and biomedical sciences. Behav Res Methods 2007; 39:175-191.

55 Little, Roderick JA: A Test of missing completely at random for multivariate data with missing values. J Am Stat Assoc 1988;83: 1198-1202.

56 Spielberger CD: State-Trait Anxiety Inventory; in The Corsini Encyclopedia of Psychology. Hoboken, Wiley, 2010.

57 Hayes AF: Introduction to Mediation, Moderation, and Conditional Process Analysis: a Regression-Based Approach. New York, Guilford Press, 2013.

58 Landerholm EJ, Scriven G: A comparison of mother and father interaction with their sixmonth-old male and female infants. Early Child Dev Care 1981;7:317-328.

59 Freedman D: Human Infancy, an Evolutionary Perspective. New York, Halsted Press, 1974.

60 Lutchmaya S, Baron-Cohen S: Human sex differences in social and non-social looking preferences, at 12 months of age. Infant Behav Dev 2002;25:319-325.

61 Connellan J, Baron-Cohen S, Wheelwright S, Batki A, Ahluwalia J: Sex differences in human neonatal social perception. Infant Behav Dev 2000;23:113-118.

-62 Bearman SK, Stice E, Chase A: Evaluation of an intervention targeting both depressive and bulimic pathology: a randomized prevention trial. Behav Ther 2003;34:277-293.

63 Costello EJ, Mustillo S, Erkanli A, Keeler G, Angold A: Prevalence and development of psychiatric disorders in childhood and adolescence. Arch Gen Psychiatry 2003;60:837844

64 Leeb RT, Rejskind FG: Here's looking at you, kid! A longitudinal study of perceived gender differences in mutual gaze behavior in young infants. Sex Roles 2004:50:1-5.

65 Montirosso R, Borgatti R, Trojan S, Zanini R, Tronick E: A comparison of dyadic interactions and coping with still-face in healthy preterm and full-term infants. Br J Dev Psychol 2010;28:347-368.

66 Falkei P, Wittchen H: Diagnostisches und statistisches Manual psychischer Störungen, DSM-5, ed 1. Göttingen, Hogrefe, 2014

- 67 Ponder KL, Salisbury A, McGonnigal B, Laliberte A, Lester B, Padbury JF: Maternal depression and anxiety are associated with altered gene expression in the human placenta without modification by antidepressant use: implications for fetal programming. Dev Psychobiol 2011;53:711-723. 